Journal of Applied Analysis

Vol. 14, No. 2 (2008), pp. 227-238

\title{
UNIFORM ALGEBRAS IN THE CANTOR AND BAIRE SPACES
}

\author{
A. NOWIK ${ }^{*}$ and P. REARDON ${ }^{\dagger}$ \\ Received June 20, 2007 and, in revised form, March 23, 2008
}

\begin{abstract}
For each pointclass $\Gamma \subseteq \mathcal{P}\left(2^{\omega}\right)$ define $U[\Gamma]$ as the collection of all $X \subseteq 2^{\omega}$ such that the preimage $f^{-1}(X)$ belongs to $\Gamma$ for each continuous $f: 2^{\omega} \rightarrow 2^{\omega}$. We study the properties of and possible relationships among the classes $U[\Gamma]$, where $\Gamma$ ranges over the $\sigma$-algebras $(l),(m)$, the completely Ramsey sets, and the sets with the Baire property. We also prove some results about cardinal coefficients of $U[\Gamma]$ for the general case of Marczewski-Burstin representable $\sigma$-algebras $\Gamma$. We finish by posing some unsolved problems.
\end{abstract}

\section{PREFACE}

In the early 1970's Fred Galvin and Karel Prikry in [11] defined two subcollections of the Ramsey sets that form $\sigma$-algebras; the Ramsey sets do not ([9]). At first, both collections were called the completely Ramsey sets. The article [11] is concerned with the collection that later became known as

2000 Mathematics Subject Classification. Primary: 03E15, 03E40; Secondary: 26A15, $28 \mathrm{E} 15$.

Key words and phrases. Ramsey set, Laver tree, superperfect tree, tree forcing, Marczewski-Burstin representation.

* Partially supported by grant BW/5100-5-0201-6.

${ }^{\dagger}$ Partially supported by an ORF grant from SE Oklahoma State University.

ISSN 1425-6908 (C) Heldermann Verlag. 
the uniformly completely Ramsey sets, while Silver in [22] studied the other $\sigma$-algebra which is still referred to as the class of completely Ramsey sets.

Definition 1.1. A set $X \subseteq 2^{\omega}$ is

1. Ramsey if there exists $A \in[\omega]^{\omega}$ such that $[A]^{\omega} \subseteq X$ or $[A]^{\omega} \cap X=\emptyset$;

2. completely Ramsey $(C R)$ if for every $A \in[\omega]^{\omega}$ and $s \in[\omega]^{<\omega}$ such that $\max (s)<\min (A)$, there exists $B \in[A]^{\omega}$ such that $[s, B] \subseteq X$ or $[s, B] \cap X=\emptyset$, where $[s, B]=\left\{x \in[\omega]^{\omega}: s \subset x \subseteq s \cup B\right\}$;

3. uniformly completely Ramsey $(U C R)$ if for every continuous function $f: 2^{\omega} \rightarrow 2^{\omega}, f^{-1}(X)$ is Ramsey, and

4. uniformly completely Ramsey null $\left(U C R_{0}\right)$ if every subset of $X$ belongs to UCR.

Darji in [10] showed that "Ramsey" may be replaced by "completely Ramsey" in the definition of UCR. Thus UCR is the closure of CR under continuous preimage. It is interesting to consider the general case. So suppose that $\Gamma$ is a pointclass over $2^{\omega}$. Let us define

$$
U[\Gamma]=\left\{X \subseteq 2^{\omega}: \forall \underset{f:{ }_{f \text { cont. }} \omega \rightarrow}{ }\left(f^{-1}(X) \in \Gamma\right)\right\},
$$

and let $H[\Gamma]$ denote the hereditary ideal associated with $\Gamma$. Notice that the "operator" $U[\cdot]$ is idempotent: $U[U[\Gamma]]=U[\Gamma]$. Moreover, if $\Gamma$ is a $\sigma$-algebra, then so is $U[\Gamma]$. We can express our previous notions in this language as follows:

Observation 1.2. $U[C R]=U C R$ and $H[U[C R]]=U C R_{0}$.

Let us define also the Borel variant of the "operator" $U[\cdot]$.

$$
U_{\mathcal{B}}[\Gamma]=\left\{X \subseteq 2^{\omega}: \underset{f:{ }^{2}{ }^{\omega} \rightarrow 2^{\omega}}{ }\left(f^{-1}(X) \in \Gamma\right)\right\} .
$$

Although the study of $U[\Gamma]$ is the primary focus of this paper, it will occasionally be useful to consider the smaller class $U_{\mathcal{B}}[\Gamma]$ for two reasons: either because we can prove a stronger result about the smaller class, or because we can only prove a weaker result about the smaller class. Other than that however, we will restrict our attention to $U[\Gamma]$.

\section{Notation}

Our notation for the most part is standard (see [16]). We consider as the base space the Cantor space $2^{\omega}$, although some of our investigations can also be considered in the space $\mathbb{R}$. By $\omega^{\uparrow \omega}, \omega^{\uparrow<\omega}, \omega^{\uparrow \leq \omega}$, and $[\omega]^{\omega}$ we denote, respectively, the strictly increasing functions from $\omega^{\omega}$, the strictly increasing functions from $\bigcup_{n \in \omega} \omega^{n}$, the sum $\omega^{\uparrow \omega} \cup \omega^{\uparrow<\omega}$, and the infinite 
subsets of $\omega$. We will often think of $\omega^{\uparrow \leq \omega}$ and $[\omega]^{\omega}$ as subsets of $2^{\omega}$, and in those cases will conflate a sequence $s \in \omega^{\uparrow \leq \omega}$ with $\phi(\operatorname{ran}(s))$, and $s \subseteq \omega$ with $\phi(s)$, where $\phi: \omega \rightarrow 2$ is the characteristic function.

Let Lebesgue denote the collection of all Lebesgue measurable sets, Baire the collection of all sets with the Baire property, $\mathcal{N}$ the $\sigma$-ideal of sets with Lebesgue measure zero, and $\mathcal{M}$ the $\sigma$-ideal of meager sets.

If $T \subseteq \omega^{\uparrow<\omega}$ and $\sigma \in \omega^{\uparrow<\omega}$, then $\operatorname{succ}_{T}(\sigma)=\{\tau \in T: \sigma \subseteq$ $\tau$ and length $(\tau)=$ length $(\sigma)+1\}$. If $\mathcal{I}$ and $\mathcal{J}$ are two $\sigma$-ideals, then we write $\mathcal{I} \perp \mathcal{J}$ iff there exists $X \in \mathcal{I}$ such that $2^{\omega} \backslash X \in \mathcal{J}$. For example, we have $\mathcal{M} \perp \mathcal{N}$. To avoid trivial cases we always assume that a $\sigma$-algebra $\mathcal{A}$ contains the Borel subsets of $2^{\omega}$. In this case the $\sigma$-algebra $U[\mathcal{A}]$, and hence the $\sigma$-ideal $H[U[\mathcal{A}]]$, contain all singletons.

\section{Definitions}

Definition 3.1. A set $X$ in a topological space is

1. always of the first category $(A F C)$ if it is meager relative to every perfect set;

2. universally meager $(\mathcal{U M}$ or $\overline{A F C})$ if it does not contain a continuous and one-to-one image of a set of second category (see [13], [14], or [23]);

3. meager additive $\left(\mathcal{M}^{*}\right)$ if $M+X$ is meager for every meager set $M$ (see $[4])$.

It was proved in [23] that a set $X$ is universally meager if and only if each Borel isomorphic image of $X$ in $\mathbb{R}$ is meager, and that a product of two universally meager sets is universally meager. Notice also that $\mathcal{U M} \subseteq A F C$.

Suppose that $\mathcal{F}$ is an arbitrary family of nonempty subsets of a set $\mathcal{X}$. The following collections of sets were considered in many papers (for example see [1], [2], or [21]).

$$
\mathcal{S}(\mathcal{F})=\left\{X \subseteq \mathcal{X}: \forall_{P \in \mathcal{F}} \exists_{Q \in \mathcal{F}}(Q \subseteq P \cap X) \text { or }(Q \subseteq P \backslash X)\right\},
$$

and

$$
\mathcal{S}_{0}(\mathcal{F})=\left\{X \subseteq \mathcal{X}: \forall_{P \in \mathcal{F}} \exists_{Q \in \mathcal{F}}(Q \subseteq P \backslash X)\right\} .
$$

We will assume throughout that $\mathcal{X}=2^{\omega}$.

Definition 3.2. A pair $(\mathcal{A}, \mathcal{I})$ (where $\mathcal{A}$ is a $\sigma$-algebra and $\mathcal{I}$ is a $\sigma$-ideal) has a Marczewski-Burstin representation (MB representation) if there exists a family of sets $\mathcal{F}$ such that $\mathcal{A}=\mathcal{S}(\mathcal{F})$ and $\mathcal{I}=\mathcal{S}_{0}(\mathcal{F})$. In this case we let $\mathcal{A}_{0}$ denote the ideal $\mathcal{S}_{0}(\mathcal{F})$. We call the MB representation inner if $\mathcal{F} \subseteq \mathcal{S}(\mathcal{F})$. 


\section{Definition 3.3.}

1. A nonempty tree $T \subseteq 2^{<\omega}$ is called perfect if $\forall_{\sigma \in T} \exists_{\tau, \tau^{\prime} \in T}(\tau \perp$ $\tau^{\prime}$ and $\left.\sigma \subseteq \tau \cap \tau^{\prime}\right)$

2. a nonempty tree $T \subseteq \omega^{<\omega}$ is called superperfect if $\forall_{\sigma \in T} \exists_{\tau \in T}(\sigma \subseteq$ $\tau$ and $\left.\left|\operatorname{succ}_{T}(\tau)\right|=\aleph_{0}\right)$, and

3. a nonemtpy tree $T \subseteq \omega^{<\omega}$ is called Laver if $\forall_{\sigma \in T}$ (stem $(T) \subseteq \sigma \Rightarrow$ $\left.\left|\operatorname{succ}_{T}(\sigma)\right|=\aleph_{0}\right)$.

We let $(\ell),(m)$, and $(s)$ denote, respectively, the $\sigma$-algebras $S\left(\mathcal{F}_{\mathbb{L}}\right)$, $S\left(\mathcal{F}_{\mathbb{M}}\right)$, and $S\left(\mathcal{F}_{\mathbb{S}}\right)$, where $\mathcal{F}_{\mathbb{L}}=\left\{[T]: T \subseteq \omega^{\uparrow<\omega}\right.$ is a Laver tree $\}$, $\mathcal{F}_{\mathbb{M}}=\left\{[T]: T \subseteq \omega^{\uparrow<\omega}\right.$ is a superperfect tree $\}$, and $\mathcal{F}_{\mathbb{S}}=\{[T]: T \subseteq$ $2^{<\omega}$ is a perfect tree $\}$. (The fact that $(\ell)$ and $(m)$ are countably additive is well-known. See [18] for example.)

Let us notice that $(\mathcal{A}, H[\mathcal{A}])$ has an inner MB representation whenever $\mathcal{A} \in\{$ Lebesgue, Baire, $C R,(\ell),(m),(s)\}$. Indeed, for all but the first two classes this follows immediately from the definition. The pairs (Lebesgue, $\mathcal{N}$ ) and $($ Baire, $\mathcal{M}$ ) have inner MB representations for $\mathcal{F}$ equal to, respectively, the family of positive measure perfect sets (see [8]), and the family of all non-meager $G_{\delta}$ sets (see [7]).

\section{Measure And CATEGORY}

This section is devoted to the case of two classical $\sigma$-algebras: Baire and Lebesgue. Let us start with the following:

Theorem 4.1. The following are equivalent for any set $X$.

1. $X$ is a universal measure zero set.

2. $X \in H\left[U_{\mathcal{B}}[\right.$ Lebesgue $\left.]\right]$.

Proof. Assume that $X$ is a universal measure zero set, $f: 2^{\omega} \rightarrow 2^{\omega}$ is Borel, and define $X_{1}=\left\{y \in 2^{\omega}: f^{-1}(y) \notin \mathcal{N}\right\}$. Since $\left|X_{1}\right| \leq \aleph_{0}$, we have that $f^{-1}\left(X_{1}\right)$ is Borel. Consider the measure $\mu$ vanishing on singletons and defined on the Borel subsets of $2^{\omega}$ given by:

$$
\mu(B)=\lambda\left(f^{-1}\left(B \backslash X_{1}\right)\right),
$$

where $\lambda$ denotes as usual Lebesgue measure. Then since $X$ has universal measure zero, we conclude that there is a Borel set $B \subseteq 2^{\omega}$ such that $\mu(B)=0$ and $X \subseteq B$. Hence $f^{-1}(X) \backslash f^{-1}\left(X \cap X_{1}\right) \subseteq f^{-1}\left(B \backslash X_{1}\right)$ and it follows that $\lambda\left(f^{-1}\left(X \backslash X_{1}\right)\right) \leq \lambda\left(f^{-1}\left(B \backslash X_{1}\right)\right)=\mu(B)=0$. Therefore, $f^{-1}(X)$ is Lebesgue measurable since $\left|X \cap X_{1}\right| \leq \aleph_{0}$. The proof of the opposite case follows from the classical result that any two Borel measures on the Cantor set vanishing on singletons are Borel isomorphic (see Theorem 17.41 in [16]). 
We do not know whether the theorem is still true with $H[U[$ Lebesgue $]]$ in place of $H\left[U_{\mathcal{B}}[\right.$ Lebesgue $\left.]\right]$.

The situation in the "Baire" case is much harder. Let us cite the following result (see [24, Theorem 1.2]) which completely solves the Baire case and strongly improves our previous results on this topic:

Theorem 4.2. $H\left[U_{\mathcal{B}}[\right.$ Baire $\left.]\right]=\mathcal{U M}$.

\section{5. $\sigma$-FiELDS ASSOCIATED WITH TREE FORCINGS}

Definition 5.1 (see [6]). A pointclass $\Gamma$ over a topological space $\mathcal{X}$ is said to be topologically reasonable (over $\mathcal{X}$ ) if it is closed under preimages by continuous functions from $\mathcal{X}$ to $\mathcal{X}$, and has the property that $X \cap Q \in \Gamma$ for every $X \in \Gamma$ and every closed $Q \subseteq \mathcal{X}$.

We begin with the following easy proposition.

Proposition 5.2. Suppose that $\Gamma$ is a pointclass over $2^{\omega}$ that contains the Borel sets and is closed under finite intersections. Then $U[\Gamma]$ is topologically reasonable over $2^{\omega}$.

Proof. $U[\Gamma]$ is obviously closed under continuous preimage. So suppose that $X \in U[\Gamma]$ and $Q \subseteq 2^{\omega}$ is closed. Suppose also that $f: 2^{\omega} \rightarrow 2^{\omega}$ is a continuous function. Then $f^{-1}(X \cap Q)=f^{-1}(X) \cap f^{-1}(Q)$. But $f^{-1}(Q)$ is Borel and thus belongs to $\Gamma$.

Corollary 5.3. Any class among $U C R, U[(\ell)], U[(m)]$, and $U[$ Baire $]$ is topologically reasonable over $2^{\omega}$.

Proof. Each of the $\sigma$-algebras $C R,(\ell),(m)$, and Baire contains the Borel sets.

The following result from [6] is crucial in our proofs of the relations from Figure 1:

Lemma 5.4. Let $\Gamma$ be a topologically reasonable pointclass. Then one has the following implications:

1. $\Gamma \subseteq(\ell) \Longrightarrow \Gamma \subseteq(m) \Longrightarrow \Gamma \subseteq(s)$, and

2. $\Gamma \subseteq$ Baire $\Longrightarrow \Gamma \subseteq(m)$. 
Therefore we have as an immediate consequence the diagram in Figure 1 which is a "uniform" version of the diagram following Corollary 3.5 of [6].

Let us prove for example:

Corollary 5.5. $U[(\ell)] \subseteq U[(m)]$.

Proof. It suffices to show that $U[(\ell)] \subseteq(m)$. Since the class $U[(\ell)]$ is topologically reasonable by Corollary 5.3 and by the implication $\Gamma \subseteq(\ell) \Longrightarrow$ $\Gamma \subseteq(m)$ from Lemma 5.4, we conclude that $U[(\ell)] \subseteq(m)$.

We can prove the other implications shown in Figure 1 and Figure 2 by the same method (except the inclusion $\mathcal{U M} \subseteq H[U[$ Baire $]]$ which follows immediately from Theorem 4.2.)

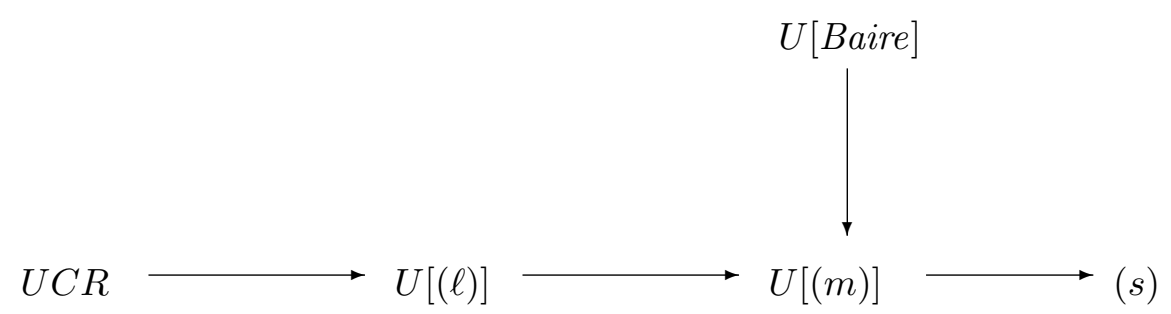

FiguRE 1. Uniform classes

Assuming the Continuum Hypothesis, all of the classes from Figure 2 contain a set of size $2^{\omega}$, namely a Sierpiński set. Let us recall the definition.

Definition 5.6. A set $X$ is Sierpinski if $X$ has cardinality continuum and the intersection of $X$ with any set from $\mathcal{N}$ is countable.

Example 5.7 (CH). Every Sierpiński set belongs to any class from Figure 2 .

Proof. Under the Continuum Hypothesis, there exists a Sierpiński set, and every Sierpiński set is $U C R_{0}$ (see [10]) and $\mathcal{U M}$ (see [13]).

The next two examples show, modulo the Continuum Hypothesis, that the inclusions indicated in Figure 2 are the only ones possible. 


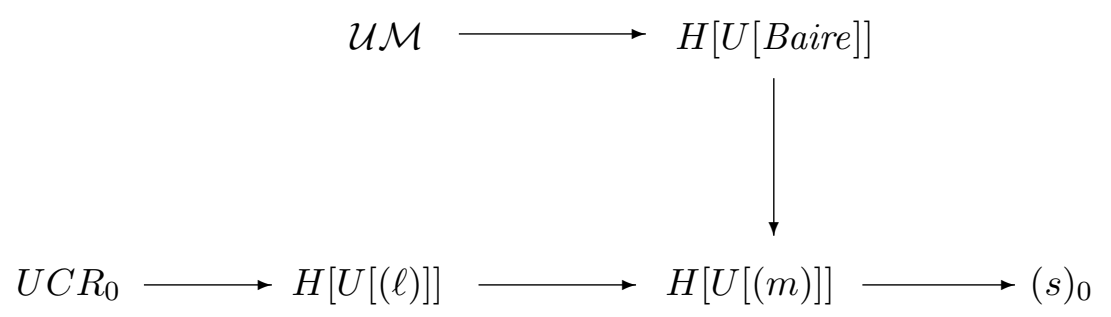

Figure 2. Hereditary classes

Example 5.8 (CH). There exists a set $X \subseteq 2^{\omega}$ such that $X$ belongs to $\mathcal{U M}$ but $X$ does not belong to $H[U[(\ell)]]$.

Proof. Assume the Continuum Hypothesis. Let $X \subseteq 2^{\omega}$ be such that $X \in \mathcal{M}^{*}$ and $X \notin(\ell)_{0}$ (Theorem 3.6 from [17]). Modifying the proof of the Proposition 3.4 from [17], we can obtain $\mathcal{M}^{*} \subseteq \mathcal{U} \mathcal{M}$, and it is easily verified that $H[U[(\ell)]] \subseteq(\ell)_{0}$.

Definition 5.9. A set $X$ is Luzin if $X$ has cardinality continuum and the intersection of $X$ with any set from $\mathcal{M}$ is countable.

Example 5.10 (CH). Every Luzin set belongs to any class in the lower row of the diagram in Figure 2, and belongs to no class in the upper row.

Proof. The Continuum Hypothesis implies there is a Luzin set, and every Luzin is $U C R_{0}$ by Theorem 9 of [10], and is not $A F C$ so can't be in $H[U[$ Baire $]]$.

\section{A separation theorem for MB representable algebras}

Lemma 6.1. If $\mathcal{A}$ has an inner $M B$ representation and add $\left(\mathcal{A}_{0}\right)=\kappa$, then $\operatorname{add}(U[\mathcal{A}])=\kappa$.

Proof. Assume the hypotheses, write $\mathcal{A}=\mathcal{S}(\mathcal{F})$, and fix $\gamma<\kappa$. First we show that $Y=\bigcup_{\alpha<\gamma} Y_{\alpha} \in \mathcal{A}$ whenever $Y_{\alpha} \in \mathcal{A}$ for all $\alpha<\gamma$. Notice that this claim was proved in [21] (see Theorem 2.2) for the case $\kappa=\omega_{0}$. For the sake of completeness we include the proof for the general case. Suppose 
that $P \in \mathcal{F}$ and that there exist $\alpha<\gamma$ and $Q \in \mathcal{F}$ such that $Q \subseteq Y_{\alpha} \cap P$. Then $Q \subseteq Y \cap P \subseteq X$.

On the other hand, suppose that no member of $\mathcal{F}$ lies in $Y_{\alpha} \cap P$ for any $\alpha<\gamma$. The MB representation is inner so $P \in \mathcal{A}$, and this implies that $Y_{\alpha} \cap P \in \mathcal{A}_{0}$. It follows that there is some $Q \in \mathcal{F}$ such that $Q \subseteq P$ and $Q \subseteq P \backslash(Y \cap P) \subseteq 2^{\omega} \backslash X$, whence $Y \in \mathcal{A}$.

Suppose $\left\{X_{\alpha}: \alpha<\gamma\right\} \subseteq U[\mathcal{A}]$ and $f$ is continuous. Then

$$
f^{-1}\left(\bigcup_{\alpha<\gamma} X_{\alpha}\right)=\bigcup_{\alpha<\gamma} f^{-1}\left(X_{\alpha}\right)
$$

and $f^{-1}\left(X_{\alpha}\right) \in \mathcal{A}$ for all $\alpha<\gamma$. It follows that $\bigcup_{\alpha<\gamma} X_{\alpha} \in U[\mathcal{A}]$.

Now let us formulate the main theorem which is a useful tool to obtain counterexamples in this setting.

Theorem 6.2. Suppose that $\mathcal{F}$ and $\mathcal{G}$ are collections of uncountable Borel sets, and put $\mathcal{A}=\mathcal{S}(\mathcal{F})$ and $\mathcal{B}=\mathcal{S}(\mathcal{G})$. Assume that

1. $\operatorname{add}\left(\mathcal{A}_{0}\right)=\operatorname{add}\left(\mathcal{B}_{0}\right)=\mathfrak{c}$;

2. $\Sigma_{1}^{1} \subseteq \mathcal{B}$, and

3. for every continuous function $f: 2^{\omega} \rightarrow 2^{\omega}$ and for every $P \in \mathcal{G}$ there exists $Q \in \mathcal{G}$ such that $Q \subseteq P$ and $f(Q) \in \mathcal{A}_{0}$.

Then there exists $X \in U[\mathcal{B}] \backslash \mathcal{A}$.

Proof. At first notice that since $|\mathcal{F}| \leq \mathfrak{c}$ and $\forall P \in \mathcal{F}(|P|=\mathfrak{c})$, we may conclude that $\mathcal{A}_{0}=H[\mathcal{A}]$. Fix enumerations $\left(L_{\alpha}: \alpha<\mathfrak{c}\right)$ of all elements of $\mathcal{F}$, and $\left((M, f)_{\alpha}: \alpha<\mathfrak{c}\right)$ of all pairs $(M, f)$ where $M \in \mathcal{G}$ and $f: 2^{\omega} \rightarrow 2^{\omega}$ is continuous.

At stage $\psi<\mathfrak{c}$, suppose that we have constructed sequences $\left\{X_{\alpha}: \alpha<\psi\right\}$ and $\left\{Y_{\alpha}: \alpha<\psi\right\}$ such that

$$
\begin{gathered}
X_{0} \subseteq X_{1} \subseteq \cdots \subseteq X_{\alpha} \subseteq \cdots \text { and } \\
Y_{0} \subseteq Y_{1} \subseteq \cdots \subseteq Y_{\alpha} \subseteq \cdots,
\end{gathered}
$$

where $X_{\alpha}$ and $Y_{\alpha}$ are disjoint subsets of $2^{\omega}$ and belong to $U[\mathcal{B}] \cap \mathcal{A}_{0}$. Define $X_{\psi}^{*}=\bigcup_{\alpha<\psi} X_{\alpha}$, and $Y_{\psi}^{*}=\bigcup_{\alpha<\psi} Y_{\alpha}$. By Lemma 6.1 and our assumption that add $\left(\mathcal{A}_{0}\right)=\mathfrak{c}, X_{\psi}^{*}$ and $Y_{\psi}^{*}$ are again in $U[\mathcal{B}] \cap \mathcal{A}_{0}$.

Write $(M, f)_{\psi}=(\hat{M}, \hat{f})$ and notice that $\hat{f}^{-1}\left(Y_{\psi}^{*}\right) \in \mathcal{B}$. This implies there is some $N_{\psi} \in \mathcal{G}$ such that $N_{\psi} \subseteq \hat{f}^{-1}\left(Y_{\psi}^{*}\right)$ or $N_{\psi} \cap \hat{f}^{-1}\left(Y_{\psi}^{*}\right)=\emptyset$. The continuous image of a Borel set is $\Sigma_{1}^{1}$, and considering (3) in the hypotheses we may assume without loss of generality that $\hat{f}\left(N_{\psi}\right) \in \Sigma_{1}^{1} \cap \mathcal{A}_{0} \subseteq U[\mathcal{B}] \cap \mathcal{A}_{0}$. Thus $\hat{f}^{-1}\left(N_{\psi}\right) \subseteq Y_{\psi}^{*}$ or $\hat{f}^{-1}\left(N_{\psi}\right) \cap Y_{\psi}^{*}=\emptyset$. Set

$$
X_{\psi}^{\prime}=X_{\psi}^{*} \cup\left(\hat{f}\left(N_{\psi}\right) \backslash Y_{\psi}^{*}\right),
$$


and put $Y_{\psi}^{\prime}=Y_{\psi}^{*}$. Notice that $X_{\psi}^{*}, Y_{\psi}^{*}$, and $\hat{f}\left(N_{\psi}\right) \backslash Y_{\psi}^{*}$ all belong to $U[\mathcal{B}] \cap \mathcal{A}_{0}$, hence so do $X_{\psi}^{\prime}$ and $Y_{\psi}^{\prime}$.

For all $\alpha<\psi$ we have $X_{\alpha}, Y_{\alpha} \in \mathcal{A}_{0}$, so $\bigcup_{\alpha<\psi}\left(X_{\alpha} \cup Y_{\alpha}\right) \in \mathcal{A}_{0}$ as well. Because of this we may choose $x_{\psi} \neq y_{\psi}$ such that $x_{\psi}, y_{\psi} \in L_{\psi} \backslash \bigcup_{\alpha<\psi}\left(X_{\alpha} \cup\right.$ $\left.Y_{\alpha}\right)$. Define $X_{\psi}=X_{\psi}^{\prime} \cup\left\{x_{\psi}\right\}$ and $Y_{\psi}=Y_{\psi}^{\prime} \cup\left\{y_{\psi}\right\}$, and note that $X_{\psi} \cap Y_{\psi}=\emptyset$. Finally, define $X=\bigcup_{\psi<\mathfrak{c}} X_{\psi}$. Observe that $L_{\alpha}$ contains a point of $X$ and a point of $X^{c}$ for every $\alpha<\mathfrak{c}$, hence $X \notin \mathcal{A}$.

To see that $X \in U[\mathcal{B}]$, fix $\psi<\mathfrak{c}$ and write $(M, f)_{\psi}=(\hat{M}, \hat{f})$. Then $\hat{f}\left(N_{\psi}\right) \subseteq X_{\psi}^{\prime} \subseteq X$, or $\hat{f}\left(N_{\psi}\right) \subseteq Y_{\psi}^{\prime}$. But this means that $\hat{f}\left(N_{\psi}\right) \cap X=\emptyset$ because $X \cap Y_{\psi}^{\prime}=\emptyset$. Since $N_{\psi} \subseteq \hat{M}$, we conclude that $X \in U[\mathcal{B}] \backslash \mathcal{A}$.

The foregoing discussion leads us to define two properties of MB represented algebras and ideals as follows. Suppose that $\mathcal{A}$ and $\mathcal{B}$ have inner Marczewski-Burstin representations $\mathcal{A}=\mathcal{S}(\mathcal{F})$ and $\mathcal{B}=\mathcal{S}(\mathcal{G})$. Consider the following two conditions:

$(\dagger)$ : for all continuous $f: 2^{\omega} \rightarrow 2^{\omega}$ and for every $P \in \mathcal{G}$, there exists some $Q \in \mathcal{G}$ such that $Q \subseteq P$ and $f(Q) \in \mathcal{A}_{0}$; and

$(\ddagger)$ : for every continuous $f: 2^{\omega} \rightarrow 2^{\omega}$, there exists some $X \in \mathcal{A}_{0}$ such that $2^{\omega} \backslash f^{-1}(X) \in \mathcal{B}_{0}$.

Then $(\ddagger)$ implies $(\dagger)$ and the condition $(\dagger)$ is exactly the property from Theorem 6.2. In the meantime, the condition $(\ddagger)$ implies the relation of "orthogonality" of the $\sigma$-ideals $\mathcal{A}_{0}$ and $\mathcal{B}_{0}$, i.e. condition $(\ddagger)$ implies that $\mathcal{A}_{0} \perp \mathcal{B}_{0}$.

The remainder of this section is devoted to proving that condition $(\dagger)$ holds for any two $\sigma$-algebras in the bottom row of Figure 2, except for the pair $U C R$ and $U[(\ell)]$, which we cannot solve. First we show that $(\dagger)$ holds when $\mathcal{A}=(m)$ and $\mathcal{B}=(s)$. Let us denote by $\leq^{*}$ the standard eventually dominating ordering of the space $\omega^{\omega}: f \leq^{*} g \Longleftrightarrow \exists_{N<\omega} \forall_{n>N}(f(n) \leq$ $g(n))$.

Lemma 6.3 ([15]). Let $X \subseteq \omega^{\omega}$ be an analytic set (i.e. $\left.X \in \Sigma_{1}^{1}\right)$. Then the following are equivalent:

1. There exists a superperfect tree $T$ such that $[T] \subseteq X$.

2. $X$ is unbounded in $\left(\omega^{\omega}, \leq^{*}\right)$.

Theorem 6.4. For every continuous $f: 2^{\omega} \rightarrow 2^{\omega}$ and every perfect tree $M$, there exists a perfect tree $N \subseteq M$ such that $f([N]) \in(m)_{0}$.

Proof. The proof is very easy. Indeed, suppose that $f: 2^{\omega} \rightarrow 2^{\omega}$ is continuous and $M$ is a perfect tree. If there exists $y \in[\omega]^{<\omega}$ such that $\left|f^{-1}(y) \cap[M]\right|>\aleph_{0}$ then $f^{-1}(y)$ contains a perfect set $[N]$ for some perfect 
tree $N$, and in that case $f([N]) \cap[\omega]^{\omega}=\emptyset$ which implies that $f([N]) \in(m)_{0}$. On the other hand, suppose that $\forall_{y \in \omega<\omega}\left(\left|f^{-1}(y) \cap[M]\right| \leq \aleph_{0}\right)$, then choose a perfect tree $N \subseteq M$ such that $f([N]) \subseteq[\omega]^{\omega}$. Since $f([N])$ is compact it is bounded in $\left(\omega^{\uparrow \omega}, \leq^{*}\right)$. Therefore $f([N]) \in(m)_{0}$ by Lemma 6.3.

Corollary 6.5. Assume Martin's Axiom. Then $U[(m)]$ is a proper subset of $U[(s)]=(s)$.

Proof. Let $\mathcal{A}=\mathcal{S}\left(\mathcal{F}_{\mathbb{M}}\right)=(m)$, and $\mathcal{B}=\mathcal{S}\left(\mathcal{F}_{\mathbb{S}}\right)=(s)$. We check the hypotheses of Theorem 6.2. Condition (1) follows from [12], Condition (2) is well-known, and Condition (3) is Theorem 6.4.

The case when $\mathcal{A}=(\ell)$ and $\mathcal{B}=(m)$ is not quite so straightforward.

Theorem 6.6. For every continuous function $f: 2^{\omega} \rightarrow 2^{\omega}$ and every superperfect tree $M \subseteq \omega^{\uparrow<\omega}$, there exists a superperfect tree $N \subseteq M$ such that $f([N]) \in(\ell)_{0}$.

Proof. At first notice that $[M]$ is homeomorphic to the Baire space $\omega^{\omega}$ and $[M]$ is a closed subset of the space $\omega^{\uparrow \omega}$. Then notice that for every Borel $B \subseteq[M]$ the following conditions are equivalent:

1. $B$ is contained in a $\sigma$-compact subset of $\omega^{\uparrow \omega}$.

2. $B$ is contained in a $\sigma$-compact subset of $[M]$.

3. $B$ does not contain any set of the form $\left[M_{1}\right]$ for some superperfect tree $M_{1}$.

This remark follows from Lemma 6.3 and from the fact that $[M]$ is a closed subset of $\omega^{\uparrow \omega}$.

Suppose that $f^{-1}\left(\omega^{\uparrow<\omega}\right) \cap[M]$ contains a set $\left[M_{1}\right]$ for some superperfect tree $M_{1}$. Then the proof is completed. So assume that for some superperfect tree $M_{1} \subseteq M$ we have $f\left(\left[M_{1}\right]\right) \subseteq \omega^{\uparrow \omega} \approx \omega^{\omega}$. Therefore, without loss of generality (by taking $f \uparrow\left[M_{1}\right]$ ) we can assume that $f: \omega^{\omega} \rightarrow \omega^{\omega}$ is a continuous function and we are looking for a Borel set $B \subseteq \omega^{\omega}$ which is not contained in any $\sigma$-compact subset of $\omega^{\omega}$ and such that $f(B)$ does not contain a set $[L]$ for any Laver tree $L$.

Consider two cases:

CASE 1: There is no Laver tree $L \subseteq \omega^{\uparrow<\omega}$ such that $[L] \subseteq f\left(\omega^{\omega}\right)$. Then the required condition is satisfied simply by $B=\omega^{\omega}$.

CAse 2: There exists a Laver tree $L \subseteq \omega^{\uparrow<\omega}$ such that $[L] \subseteq f\left(\omega^{\omega}\right)$. "Prune" the tree $L$ to a superperfect tree $M^{*} \subseteq L$ such that there is no Laver tree $L_{1}$ such that $\left[L_{1}\right] \subseteq\left[M^{*}\right]$. Let us consider the set $f^{-1}\left(\left[M^{*}\right]\right)$. Suppose that this set is contained in some $\sigma$-compact set $K \subseteq \omega^{\omega}$. Then we have $f^{-1}\left(\left[M^{*}\right]\right) \subseteq K$, hence $\left[M^{*}\right] \subseteq f(K)$ (since $\left.\left[M^{*}\right] \subseteq f\left(\omega^{\omega}\right)\right)$. This is however 
impossible since $f(K)$ is a $\sigma$-compact set and $M^{*}$ is a superperfect tree. Hence $f^{-1}\left(\left[M^{*}\right]\right)$ is not contained in any $\sigma$-compact subset of $\omega^{\omega}$. Put $B=$ $f^{-1}\left(\left[M^{*}\right]\right)$. Then $f(B)=f\left(f^{-1}\left(\left[M^{*}\right]\right)\right)=\left[M^{*}\right] \cap f\left(\omega^{\omega}\right)=\left[M^{*}\right]$, therefore $f(B)$ does not contain any set $\left[L_{1}\right]$ for any Laver tree $L_{1} \subseteq \omega^{\uparrow<\omega}$.

Corollary 6.7. Assume Martin's Axiom. Then $U[(\ell)]$ is a proper subset of $U[(m)]$.

Proof. Let $\mathcal{A}=\mathcal{S}\left(\mathcal{F}_{\mathbb{L}}\right)=(\ell)$ and $\mathcal{B}=\mathcal{S}\left(\mathcal{F}_{\mathbb{M}}\right)=(m)$. We check the hypotheses of Theorem 6.2. Condition (1) follows from [12], Condition (2) is a consequence of the fact that $\Sigma_{1}^{1} \subseteq C R$, (see [19],[22]), and Condition (3) is Theorem 6.6.

\section{Open PROBLEMS}

1. Does $H[U[$ Lebesgue $]]=H\left[U_{\mathcal{B}}[\right.$ Lebesgue $\left.]\right]$ ?

2. We know $([20])$ that it is consistent that no $U C R_{0}$ set has size $\mathfrak{c}$. What about the class $H[U[(m)]]$ ? Can we find in ZFC a set $X \in H[U[(m)]]$ of size $\mathfrak{c}$ ?

3. Does the condition $(\dagger)$ hold in the case $\mathcal{A}=C R=S(\mathcal{F})$ and $\mathcal{B}=(\ell)$, where $\mathcal{F}=\left\{[s, B]: s \in[\omega]^{<\omega}\right.$ and $B \in[\omega]^{\omega}$ and $\left.\max (s)<\min (B)\right\}$ ?

4. Find any set $X \in H[U[(\ell)]] \backslash U C R_{0}$.

\section{References}

[1] Balcerzak, M., Bartoszewicz, A., Rzepecka, J., Wroński, S., Marczewski fields and ideals, Real Anal. Exchange 26(2) (2000/2001), 703-715.

[2] Bartoszewicz, A., Kotlicka, E., Relationships between continuity and abstract measurability of functions, Real Anal. Exchange 31(1) (2005/2006), 73-96.

[3] Bartoszyński, T. Remarks on small sets of reals, Proc. Amer. Math. Soc. 131(2) (2003), 625-630.

[4] Bartoszyński, T., Judah, H., Borel images of sets of reals, Real Anal. Exchange 20(2) (1994/5), 536-558.

[5] Brendle, J., Strolling through paradise, Fund. Math. 148 (1995), 1-25.

[6] Brendle, J., Löwe, B., Solovay-type characterizations for forcing-algebras, J. Symbolic Logic 64 (1999), 1307-1323.

[7] Brown, J. B., Elalaoui-Talibi, H., Marczewski-Burstin-like characterizations of $\sigma$ algebras, ideals, and measurable functions, Colloq. Math. 82 (1999), 277-286.

[8] Burstin, C., Eigenschaften messbaren und nichtmessbaren Mengen, Wien Ber. 123 (1914), 1525-1551.

[9] Corazza, P., Ramsey sets, the Ramsey ideal, and other classes over R, J. Symbolic Logic 57(4) (1992), 1441-1468.

[10] Darji, U., On completely Ramsey sets, Colloq. Math. 64(2) (1993), 163-171. 
[11] Galvin, F., Prikry, K., Borel sets and Ramsey's theorem, J. Symbolic Logic 38 (1973), 193-198.

[12] Goldstern, M., Johnson, M., Spinas, O., Towers on trees, Proc. Amer. Math. Soc. 122 (1994), 557-564..

[13] Grzegorek, E., Always of the first category sets, Proceedings of the 12th Winter School on Abstract Analysis (Srni, 1984), Rend. Circ. Mat. Palermo (2) Suppl. 6 1984, 139-147.

[14] Grzegorek, E., Always of the first category sets (II), Proceedings of the 13th Winter School on Abstract Analysis (Srni, 1985), Rend. Circ. Mat. Palermo (2) Suppl. 10 (1985), 43-48.

[15] Kechris, A. S., A notion of smallness for subsets of the Baire space, Trans. Amer. Math. Soc. 229 (1977), 191-207.

[16] Kechris, A. S., Classical Descriptive Set Theory, Springer, Berlin, 1995.

[17] Kysiak, M., Nowik, A., Weiss, T., Special subsets of the reals and tree forcing notions, Proc. Amer. Math. Soc. 135 (2007), 2975-2982.

[18] Löwe, B., Uniform unfolding and analytic measurability, Arch. Math. Logic 37(8) (1998), 505-520.

[19] Mathias, A. R. D., On a generalization of Ramsey's theorem, Notices Amer. Math. Soc. 15 (1968), 931.

[20] Nowik, A., Possibly there is no uniformly completely Ramsey null set of size $2^{\omega}$, Colloq. Math. 93(2) (2002), 251-258.

[21] Pawlikowski, J., Parametrized Ellentuck theorem, Topology Appl. 37 (1990), 65-73.

[22] Silver, J., Every analytic set is Ramsey, J. Symbolic Logic 35 (1970), 60-64.

[23] Zakrzewski, P., Universally meager sets, Proc. Amer. Math. Soc. 129(6) (2001), 1793-1798.

[24] Zakrzewski, P. Universally meager sets, II, Topology Appl. 155 (2008), 1445-1449.

ANDRZEJ NOWIK

Department of Mathematics

GDAŃSK UNIVERSity

Wita Stwosza 57

80-952 Gdańsk, Poland

E-MAIL: NOWIK@MANTA.UNIV.GDA.PL
PATRICK REARDON

Department of Mathematics

SE Oklahoma State University

DURANT, OK 74701

USA

E-MAIL: PREARDON@SOSU.EDU 